\title{
Simulation and Analysis of UWB Indoor Channel Through S-V Model for User Location Detection
}

\author{
B. R. Jadhavar and T. R. Sontakke
}

\begin{abstract}
UWB has advantages of high transmission speed, great system storage, excellent multipath immunity capacity, limited power wastage and low cost, ultra-wideband technology is considered a key technology to next generation wireless communication. The channel model is generated from a modified S-V model. An ultra-wide bandwidth (UWB) signal propagation experiment is performed in a typical modern office building in order to characterize the UWB signal propagation channel. The bandwidth of the signal used in this experiment is more than one GHz. The observed channel was very slowly time varying, with the delay spread about $200 \mathrm{~ns}$ and $\mathrm{rms}$ values of up to about $50 \mathrm{~ns}$. The attenuation varied over a $60 \mathrm{~dB}$ dynamic range. Finally modified S-V model is described and used to simulate the UWB channel model. Simulation results of the model are presented. The result shows that all modulation is appropriate for both LOS and NLOS channel. Moreover, as standard of speed has been given for UWB, the communication is appropriate with high bit rates in LOS channel.
\end{abstract}

Index Terms-Channel model, Indoor LOS NLOS, MPC's, S-V Channel, UWB Model, Wireless network.

\section{INTRODUCTION}

Great strides have been made in science and technology since 19th century, when Morse transmitted first telegraphic signal on ten kilometer wire line. That was the first step toward shattering the barriers of space and time in communication between individuals. The second step was the successful deployment of radio communications at the start of $20^{\text {th }}$ century. The success of cellular radio in providing telecommunication services to the mobile and handheld portable units since 1990s has paved the way toward breaking the location barrier in telecommunications. With the advances in technologies it has become necessary to have information of location of mobile user in indoor and outdoor environment. Geographic location information can be retrieved by various techniques. Global positioning system (GPS) is effective and accurate but it works poorly in indoor and urban environment. Cellular network can be used to detect location, but because of low transmitter power and narrow bandwidth, it gives limited accuracy (in the range of few hundred meters). Less attention has been paid to the most challenging problem of locating and tracking mobile users, in-building environments and there has been no serious attempt to develop a scientific basis for location of mobile users in indoor environments.

With regard to the various media, IR (Infra-Red), RF (Radio Frequency), and Ultrasound have been used as a general medium for the positioning system. But these media

Manuscript received March 26, 2011; revised October 10, 2011.

The authors are with the Siddhant College of Engineering, Pune, India. (e-mail: brjadhavar@yahoo.co.in; trsontakke@gmail.com) are not adequate for the indoor positioning system.

Recently UWB is used in indoor applications. It is radio technology having a spectrum that occupies a bandwidth greater than $20 \%$ of the center frequency, or a bandwidth of at least $500 \mathrm{MHz}$ It has a very wide spectrum of frequencies several $\mathrm{GHz}$ in bandwidth.. In-building communications are evolving with modern network architecture. The cordless telecommunications second and fourth generations are primarily in-building communication systems, while the universal portable digital communications (UPDC) in the United States calls for a unification of the indoor and outdoor portable radio communications into an overall integrated system.

In a typical indoor portable communication system a fixed antenna is installed in an elevated position communicates with a number of portable radios inside the building. Due to reflection, refraction and scattering of radio waves by structures inside a building, the transmitted signal most often reaches the receiver by more than one path, resulting in a phenomenon known as multipath fading. The signal components arriving from indirect paths and the direct path combine and produce a distorted version of the transmitted signal. In narrow-band transmission the multipath medium causes fluctuations in the received signal amplitude and phase. In wide-band pulse transmission, [7] on the other hand, the effect is to produce a series of delayed and attenuated pulses for each transmitted pulse. The received signal is further corrupted by noise and co channel interference. Multipath fading seriously degrades the performance of communication systems operating inside buildings. Unfortunately, one can do little to eliminate multipath disturbances. However, if the multipath medium is well characterized, transmitter and receiver can be designed to "match" the channel and to reduce the effect of these disturbances. Detailed analysis of signal propagation is therefore a major requirement for successful design of indoor communication systems for indoor environment.

Therefore the use of wireless radio for indoor data or voice communications, within an office building, warehouse, a factory, large shopping complex, hospital, malls, large public gathering, airports or an apartment building, is an attractive proposition to researchers. Many times it is not convenient to carry the sensors for detection of user or object. If radio communication services are used, it would reduce wiring in a new building and would provide the flexibility of changing or creating various equipments in existing buildings without the need for expensive, time-consuming rewiring. For more number of user detection, certainly one has to go for radio communication whose technique would certainly require the knowledge of the spatial and temporal statistics of the signal attenuation, the multipath delay spread, and even the impulse response of the indoor radio channel. The 
performance of communications systems using portable radiotelephones [1], [2] will strongly depend on the attenuation characteristics of radio signals propagating into and within buildings. Scattering due to the presence of objects like wooden and steel furniture and other disturbances in the propagation medium can produce rapid signal level fluctuations from small movements of transmitter receiver or motion of the people in room [3]. Received signal powers averaged over this multipath fading often vary as a negative power of the distance between transmitter and receiver. In [4] and [5] attenuation of microwave CW signals propagating within buildings or into buildings are discussed. The feasibility of portable radio telephone systems depends on the radio signal statistics within severely attenuating buildings.. A steel shelled building exhibiting these conditions served as a test range for obtaining attenuation measurements at $900 \mathrm{MHz}$. The work of Alexander [4], at $900 \mathrm{MHz}$, is particularly useful since it gives the power law representing the signal attenuation as a function of distance for various types of building. The first multipath delay spread measurements within a building were reported recently by Devasirvatham [6]. He used a carrier at $850 \mathrm{MHz}$, bi-phase modulated with a $40 \mathrm{Mbit} / \mathrm{sec}$ maximal-length pseudo noise code, resulting in a $25 \mathrm{~ns}$ time resolution. The measurements were made in a large office building which occupies an area of $315 \times 110 \mathrm{~m}$.

We simulate and analyze a statistical model of the indoor radio channel, which has 1) enough flexibility to permit reasonably accurate fitting of the measured channel responses, 2) is simple enough to use in simulation and analysis of various indoor communications schemes, and 3) appears to be extendable (by adjusting its parameters) to represent the channel within other buildings.

As a result, studying the UWB channel characteristics, establishing the channel model are extremely important to UWB communication technology.

The testing of UWB system propagation channel has become a focal research in recent years[7]. However, many problems have not been resolved and UWB channel modeling have variances in different literatures. The reason of this phenomenon is the different measurement conditions and the deficiency of measurement data on the one hand, on the other hand is reckoning without the particular characteristics of UWB channel. A mass of experiments data showed that the UWB channel multipaths arriving in clusters, so the UWB channel model should incarnate this new peculiarity in the multipath model. In the UWB multipath model, the main parameters are excess delay, RMS delay spread, power decay profile, number of multipath components and so on. The remainder of the paper is described some typical UWB indoor channel models.

\section{BACKGROUND}

UWB characterizes transmission systems with instantaneous spectral occupancy in excess of $500 \mathrm{MHz}$ or a fractional bandwidth of more than $20 \%$. The fractional bandwidth is defined as $B / f_{c}$, where $B=f_{H}-f_{L}$ denotes the $-10 \mathrm{~dB}$ bandwidth and center frequency $f_{C}=\left(f_{L}+f_{H}\right) /$ 2 with $f_{H}$ being the upper frequency of the $-10 \mathrm{~dB}$ emission point, and $f_{L}$ the lower frequency of the $-10 \mathrm{~dB}$ emission point. Because of ultra-short duration pulse it occupy UWB spectrum.

These systems rely on ultra-short (nanosecond scale) waveforms that can be free of sine-wave carriers and do not require IF processing because they can operate at baseband. Before 2001 UWB was primarily limited to radar systems, mainly for military applications. In the mid-1980s, the FCC encouraged an entirely new type of wideband communications when it allocated the Industrial Scientific and Medical (ISM) bands for unlicensed spread spectrum and wideband communications use. This revolutionary spectrum allocation is most likely responsible for the tremendous growth in Wireless Local Area Networks (WLAN) and Wi-Fi today, as it led the communications industry to study the merits and implications of wider bandwidth communications than had previously been used for consumer applications. The first impulse system patent was awarded in 1954 and the basic concept was first described in 1960. It has been used since 1980 in military Radar applications. A substantial change occurred in 2002 when UWB was made public (by FCC). On February 14, 2002, the FCC amended the Part 15 rules which govern unlicensed radio devices to include the operation of ultra wideband (UWB) devices (Fig.1).
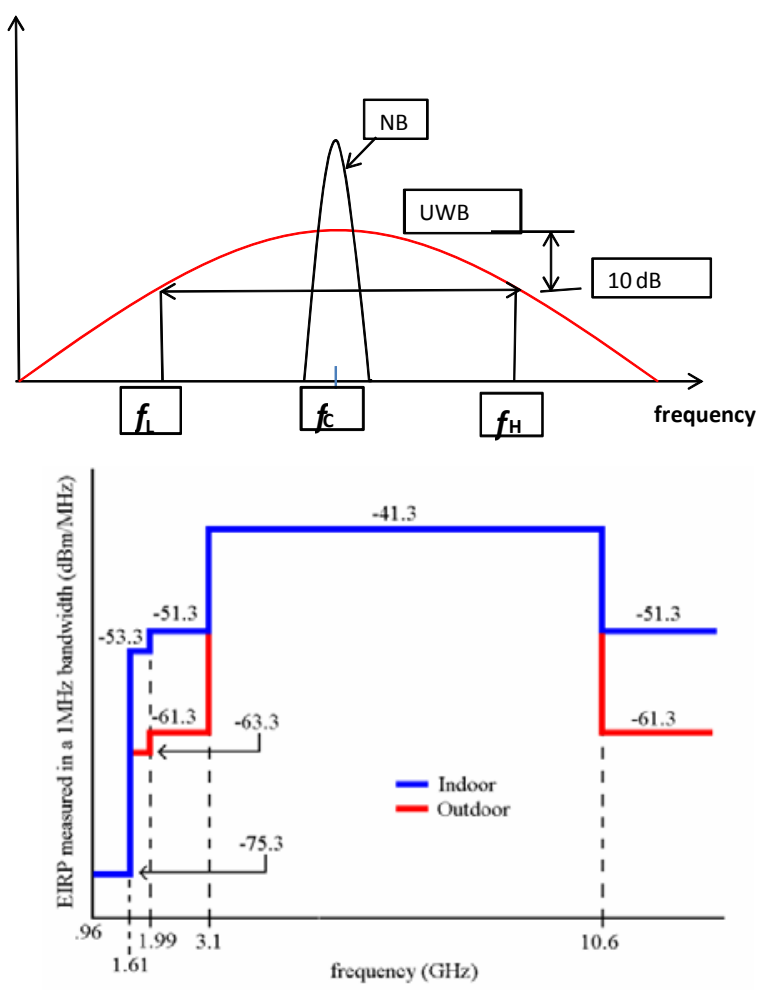

Fig. 1

\section{Propagation Mechanism}

The signal received by the receiver in communication system is a summation of both direct LOS and several multipath components. Multipath occurs due to the three basic multipath propagation mechanisms, namely reflection, diffraction and scattering of the transmitted signal. Due to this the signal received at receiver will not be exact replica of transmitted signal. The received power is generally most important parameter. All these phenomenon cause radio signal distortions and give rise to signal fading. The relative importance of these propagation mechanisms depends on the 
particular environments i.e. if there is a LOS between terminals, the reflection dominates the propagation, while if the mobile is in a heavily cluttered area with no LOS path, scattering and diffraction play major role.

\section{A. Reflection}

When radio wave propagating in one medium impinges upon another medium having different electrical properties, the wave are partially reflected, partially transmitted. If the plane wave is incident on a perfect dielectric, part of the energy is transmitted into the second medium and part of the energy is reflected back into the first medium, and there is loss of energy in absorption. The change in direction of a wave front at an interface between two dissimilar media so that the wave front returns into the medium from which it originated is called reflection. Diffraction is the spreading out of waves. It is clear that the diffraction loss increases with increasing frequency. In indoor system, diffraction is bound to be there because of complicated interior of the room.

\section{B. Scattering}

When radio wave impinges on a rough surface, the reflected energy is spread out in all direction due to scattering. The secondary waves resulting from the obstructing surface are present throughout the space and even behind the obstacle, giving rise to bending of waves around the obstacle even when LOS path does not exist between transmitter and receiver. Objects such as lamp posts, knife edged objects, furniture corners, tend to scatter energy in all directions. Here this provides additional energy to the receiver. Scattering plays a major role if the mobile is in a heavily cluttered area with NLOS path. Scattering is obvious in such a factory, hall or any other cluttered area.

\section{UWB Signals AND MOdULATION TECHNIQES}

Although UWB spectrum has a centre frequency, it is not modulated system in sense that message alters a fix frequency carrier wave. Transmitter output consists of pulses whose position, duration, amplitude or phase is altered by message. UWB have short duration and special shape (Fig.2), with $0.5 \%$ duty cycle, broadband and PSD is $10 \%$ of its peak value and is often below ambient interference. FCC mandates emission from source to be below $75 \mathrm{nW} / \mathrm{MHz}$. Hence can be operated without license. Wide variety of pulses e.g. Gaussian, nth derivative of Gaussian, chirp, Rayleigh or orthogonal wavelets can be used and choice affect BW and error performance. Number of techniques may be used with UWB systems. The potential schemes include both orthogonal and antipodal schemes.

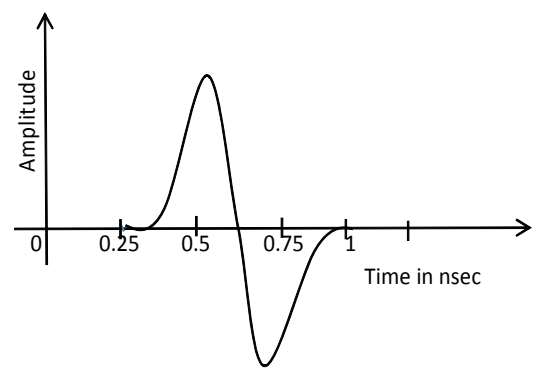

Basic pulse transmitted by Transmeter is called monocycle. It's not one cycle of a sine wave, but a unique pulse shaped by a Gaussian filter Fig. 2
By far the most common method in the literature is pulse position modulation (PPM) where each pulse is delayed or Sent in advance of a regular time backward shift in time [8]. There is variety of signal types used for UWB pulses, including Gaussian, $\mathrm{n}^{\text {th }}$ derivative of Gaussian, Chirp, and Rayleigh. The choice of the pulse width affects system error performance an bandwidth.

Let Gaussian pulse delayed by $t_{d}$

$$
p^{0}\left(t-t_{d}\right)=\frac{1}{\sqrt{2 \pi \sigma^{2}}} e^{\frac{-\left(t-t_{d}\right)^{2}}{2 \sigma^{2}}}
$$

Replacing $2 \sigma^{2}$ by $\frac{\tau^{2}}{\pi}$

And taking derivative w. r. t. time, we get Gaussian monocycle of first order [8].

$$
p^{1}\left(t-t_{d}\right)=\frac{-2 \pi\left(t-t_{d}\right)}{\tau^{2}} e^{\frac{-\pi\left(t-t_{d}\right)^{2}}{\tau^{2}}}
$$

For binary message the code for UWB pulse for OOK and PAM techniques is

$$
x_{\text {сООК }, P A M}(t)=A_{c} \sum_{k=0}^{N} a_{k} p(t-k T)
$$

$a_{k}$ is amplitude of $k^{\text {th }}$ pulse

The respective $\mathrm{N}$ number of pulses of PPM or PAM described by above equation in presence of $\mathrm{M}$ interfering signals at receiver detector input is

$v_{P P M}(t)=$

$\left[A_{c} \sum_{k=0}^{N} p\left(t-k T-d_{k} \Delta\right)\right]+\left[\sum_{j=1}^{M} \sum_{k=1}^{N} a_{j}^{k} p^{j}(t-k T-\right.$

$\left.\left.d_{k}^{j} \Delta\right)\right]$

\section{And}

$v_{P A M}(t)=\left[A_{c} \sum_{k=0}^{N} a_{k}(t-k T)\right]+\left[\sum_{j=1}^{M} \sum_{k=1}^{N} a_{k}^{j} p^{j}(t-\right.$ $\left.\left.k T-d_{k}^{j} \Delta\right)\right]$

First term is derived signal and second is summation of all signals including interference.

\section{A. Pulse amplitude modulation}

Pulse amplitude modulation (PAM)is implemented by binary pulse modulation which is presented using two antipodal Gaussian pulses. The transmitted PAM signal can be expressed as

$$
x(t)=d_{j} w_{t r}(t)
$$

where $w_{t r}$ is UWB waveform and

$$
\begin{gathered}
d_{j}=-1, j=0 \\
d_{j}=1, j=1
\end{gathered}
$$

When the pulse is transmitted, due to derivative characteristics of the antenna, the output of the transmitter can be modeled by first derivative of the pulse [9]

$$
x^{1}(t)=\lim _{d t \rightarrow 0} \frac{x(t+d t)-x(t)}{d t}
$$

and PSD of deterministic power signal is

$$
p(f)=\lim _{T \rightarrow \infty}\left\{\frac{|X(f)|^{2}}{T}\right\}
$$

where $X(f)$ is Fourier transform and

$p(f)=$ watt $/$ Hertz The normalized PSD can used to comply with FCC mask of pulse. Normalized PSD can be defined as $\quad|p(f)|=\frac{|X(f)|^{2}}{\left|X\left(f_{M}\right)\right|^{2}}$

This is to fulfill FCC rules.

\section{B. Pulse position modulation}

Pulse position modulation (PPM) makes the chosen bit be transmitted influences the position of the UWB pulse. That 
means that while bit 0 is represented by a pulse originating at the time instant 0 , bit 1 is shifted in time by the amount of $\delta$ from 0 . The PPM signal can be represented as

$$
x(t)=w_{t r}\left(t-\delta d_{j}\right)
$$

where $w_{t r}(t)$ is represented by (2). The value of $\delta$ is chosen according to the autocorrelation characteristics of the pulse. When standard PPM with orthogonal signals is implemented, the optimum value for $\delta$. ( $\left.\delta_{o p t}\right)$ will be the one which satisfies

$$
\rho\left(\delta_{\text {opt }}\right)=\int_{-\infty}^{+\infty} \mathrm{w}_{\mathrm{tr}}(\mathrm{t}) \mathrm{w}_{\mathrm{tr}}\left(\delta_{\text {opt }}+\mathrm{t}\right) \mathrm{dt}=0
$$

The theoretical performance in an additive white Gaussian noise channel can be achieved with non-overlapping orthogonal pulses, specifically, the modulation index $\delta \leq T_{c}$ [4]. If the message is in the binary form, then, the expression for PPMUWB signal is

$$
\mathrm{x}_{\mathrm{CPPM}}(\mathrm{t})=\mathrm{A}_{\mathrm{c}} \sum_{\mathrm{k}=0}^{\mathrm{N}} \mathrm{p}\left(\mathrm{t}-\mathrm{kT}-\mathrm{d}_{\mathrm{k}} \Delta\right)
$$

where $p(t)$ is UWB pulse shape, T is frame duration, $d_{k}$ represents $k^{t h}$ message bit,

In binary $d_{k}=0,1$ and $\Delta$, PPM time shift.

\section{Classification Ofuwb Channel Models}

In early years UWB was considered to be interference. It was only used for radar applications. Recently many authors have proposed propagation channel to obtain simple but reliable models. One reason for the abundance of modeling approaches is the complex phenomena encountered by a transmitted signal. The transmitted signal will usually arrive at the receiver via multipaths, where the signal encounters various propagation mechanisms. Many different types of simplifications and approximations are necessary in order to obtain a simple yet reliable model for wireless communication channel. There are many approaches try to define a radio propagation channel with a high degree of confidence. Deterministic and statistical models are the two main branches that can identify the radio channel.

\section{A. Deterministic model}

The aim of deterministic channel is to solve Maxwell's equations in either exact or approximate way. Maxwell's equations are very popular for channel prediction and network planning, which are used for ray tracing at high frequencies. These models explain the properties such as reflection, diffraction, scattering and path loss. The traditional ray tracing is used for narrowband systems. For UW channel models this has to modified as per physical environment. Hence accuracy of these model depends on accuracy of site specifications. For this accurate and detail data base of the indoor environment is essential. This is because electrical properties of the material used in building affect the signal. The electromagnetic parameters are dependent on dielectric material for building[19]. For indoor environment, indoor channel modeling usually needs availability of the three dimensional database for channel estimation. The major advantage of these models is that they offer great accuracy with site-specific results. Any site can be modeled if its physical characteristics are available, and any parameter can be calculated by adjusting these models. These models have several disadvantages such as the topographical and building data is required, which is site specific. Also, they are usually computationally intensive, especially when the environment is complex.

\section{B. Statistical model}

Stochastic channel models are much popular for simulation and testing of wireless systems. Statistical models can provide sufficiently accurate channel information and Statistical models are normally less complex than the deterministic models. This category of models has the ability to provide accurate statistical information, without the complexity of detailed deterministic approaches. These models generate channel responses that represent real propagation channel. They reflect essential properties of channel without trying to emulate exact behavior for each location. These models can be tuned to various propagation environments by setting appropriate values for channel model parameters. The main parameters of these models are path loss, delay dispersion, power delay profile, arrival times MPC's, cluster parameters, small scale fading, angular dispersion and temporal variations. These models attempt to generate channel responses that are representative of real propagation channels. Note that fixed parameter settings do not produce identical outputs on each simulation run but stochastic processes are used to create variability within a fixed environment type. A particular set of parameters might generate a representative set of propagation scenarios found in indoor environments. Statistical models may be formed based on the basic principles of wave propagation for random communications channels and by assuming statistical distribution of the channel parameters, and computing the required statistical moments from the data collected from the real-time measurements. Several statistical-based UWB channels have been proposed recently [15] [18].

\section{UWB INDOOR REFERENCE CHANNEL MODELS}

The propagation models have traditionally focused on predicting the average power received signal strength at a given distance from the transmitter as well as the variability of signal strength to a particular location. For UWB communication channel used for short distances the analysis is complex due to high frequency and large bandwidth.

\section{A. The Poisson model and its modified model}

Poisson model [11] is the pioneer UWB channel model.

This corresponds to number times an event occurs in an interval $T$ when the probability of single occurrence in a small interval $\Delta T$ is $\mu \Delta T$ The resulting Poisson frequency function is

$$
P_{I}(i)=e^{-\mu T \frac{(\mu T)^{i}}{i !}}
$$

From which, $m=\mu T, \sigma^{2}=m$ which relates to time distribution of events.

In indoor communication, suppose there is only one cluster of multipaths in impulse response, the arrived multipath components are regarded as a Poisson process. The arriving rate of the process is $\lambda$, and the path fading exponentially, the time attenuation constant is $\gamma$, the path amplitude obeys log-normal distribution and the standard variance is $\sigma$. The modified Poisson model can describe the important multipath forepart arrived components better in line-of-sight (LOS) channels. The modified Poisson model has two states S-1 and $\mathrm{S}-2$. Whenever path occurs, the mean arrival rate is increased 
or decreased by factor $\mathrm{K}$ for next $\Delta$ seconds where $\mathrm{K}$ and $\Delta$ are parameters to be chosen may be function $t_{k}$ (path arrival time) [10].

When $k=1$ or $\Delta=0$, the process is standard Poisson model. For $>1$, the probability that thee will be another path within next $\Delta$ seconds increases and it shows process is having clustering property. And for $k<1$, the incidence path decreases the probability of having another path within next $\Delta$ seconds. To handle non-stationary processes, the discrete version of modified Poisson model is used where $\mathrm{K}$ is constant and $\Delta$ is taken as fixed distance time interval.

\section{B. The $\Delta-K$ Model}

Based on the Poisson model, [14] Suzuki proposed $\Delta-K$ model. This model can better describe the multi paths arriving in clusters. If $\lambda_{i}(i=1,2,3, \ldots)$ is path occupancy rate of $i^{t h}$ bin and $\Delta$ is on timeslice, and $K$ is constant, then the probability to have path in first bin is $\lambda_{1}$. If path does not occur in bin 1 , then probability of path in bin 2 is $\lambda_{2}$. While the path does occur in bin 1, the probability of path in bin 2 is $K \lambda_{2}$. The $\Delta-K$ model introduces a new parameter $K$. If the $(n-1)^{t h}$ timeslice has one path arrived, the arrival rate of the $n^{\text {th }}$ timeslice is $k \lambda_{n}$ otherwise $\lambda_{n}$, here $\lambda_{n}$ is given by

$$
\lambda_{1}=r_{1}, \quad \lambda_{n}=\frac{r_{n}}{(K+1) r_{n-1}+1}, n \geq 2
$$

$\lambda_{n}$ is occupancy rate $n$th timeslice. When $K=1, \Delta-K$, model becomes Poisson model: when $K>1$, the channels appears in clusters; when $K<1$, the multipaths appear symmetrically. The theoretical path distribution has tendency to become flatter as the factor $\mathrm{K}$ increases. This is because the probabilities that the process has relatively small or large number of paths in interval increases as process shows clustering property. On the other hand, the peak of distribution becomes higher as $\mathrm{K}$ increases. This is because events in the interval tends to cluster around the mean value. $\mathrm{K}$ has value between 0.10 and 3.00. Also $\mathrm{K}$ increases if more bins are taken into consideration. While using the $\Delta$-K model to simulate UWB channel, the impulse response amplitude of the $n^{\text {th }}$ timeslice nears log-normal distribution, and fading exponentially with the time delay. This is given by (15)

$$
\begin{aligned}
& 20 \log _{10}^{\left(\left|\alpha_{n}\right|\right)} \propto \operatorname{Normal}\left(\mu_{n}, \sigma^{2}\right) \\
& \left|\alpha_{n}\right|=10^{\frac{p}{20}}, p \propto \operatorname{Normal}\left(\mu_{n}, \sigma^{2}\right) \\
& \left.E|| \alpha_{n}\right|^{2} \mid=\Omega_{0} e^{\frac{-T_{n}}{\Gamma}}
\end{aligned}
$$

where

$$
\mu_{\mathrm{n}}=\frac{10 \ln \left(\Omega_{0}\right)-10 \mathrm{~T}_{\mathrm{n}} / \Gamma}{\ln (10)}-\frac{\sigma^{2} \ln (10)}{20}
$$

which gives the average power of the first path, the time delay and amplitude attenuation. Although the $\Delta$-K model is improvement over standard Poisson model, for indoor application it could not best fit the measurement data of indoor UWB channel in many cases.

\section{S-V/IEEE802.15.3a model}

Saleh-Valenzuela reported the result of indoor propagation measurements between two vertically polarized omni-directional antennas located on same floor of medium sized building. The method involved averaging the square law detected pulse response while sweeping the frequency of transmitted pulses. With this method multipath components within 5 nsec. were resolvable. The result shows that: a)
Indoor channel is quassi-static or very slowly with time. b) The statistics of the channel response are independent of transmitter and receiver antenna polarization if there is no line of sight between them. They reported maximum delay spread of $100 \mathrm{nsec}$ to $200 \mathrm{nsec}$ within room and $300 \mathrm{nsec}$ in hallways. The measured rms delay spread within room had median of $25 \mathrm{nsec}$. and maximum $50 \mathrm{nsec}$. The large scale path loss with no LOS was $60 \mathrm{~dB}$, and obey the log-distance per law with exponent between three and four [11].

$$
\overline{P L}(d B)=\overline{P L}(d o)+10 n \log \left(\frac{d}{d_{0}}\right)
$$

The amplitude of received components are independent Rayleigh random variables with variances that decay exponentially with cluster decay as well as excess delay within clusters.

Afterwards, this model was modified by IEEE group which has some problems: S-V/IEEE802.15.3a can exactly reflect the propagation law of indoor NLOS environment, but its capability of indoor LOS environment is insufficient. Moreover this model cannot exactly predict multipath propagation characteristics of indoor conditions.

Saleh-Valenzuela model [12] is the universal statistic model of indoor dispersion channel impulse response. In order to perfectly inosculate with the UWB measurement data, the IEEE workgroup made some modification on the $\mathrm{S}-\mathrm{V}$ model. This can be used for NLOS channels effectively, while it can also be used for LOS channels. Because of large UWB channel bandwidth only few multipath components overlap within each resolvalable delay bin. Hence here central limit theorem cannot be applied. So also amplitude statistics are no longer Rayleigh. Saleh-Valenzuela conducted the experiment in indoor environment using RF sweep oscillator to generate $1.5 \mathrm{GHz}$. The radarlike pulses were generated and transmitted via vertically polarized antenna. The signal at various locations were observed. This pulse has the complex time domain representation

$$
x(t)=p(t) e^{j(\omega t+\emptyset)},
$$

where $p(t)$ is the baseband pulse shape, and $\omega$ is the RF angular frequency and $\varnothing$ is an arbitrary phase. The channel is represented by multiple paths or rays having real positive gains $\beta_{k}$, propagation delays $\tau_{k}$, and associated phase shifts $\theta_{k}$, where $k$ is the path index; in principle, $k$ extends from 0 to $\infty$.

When signal is transmitted by transmitter and it is picked up by receiving antenna via different paths.

Then, the complex, low-pass channel impulse response is given by [13]

$$
h(t)=\sum_{k} \beta_{k} e^{j \theta_{k}} \delta\left(t-\tau_{k}\right)
$$

where $\delta($.$) is the Dirac delta function; Because of the motion$ of people and equipment in and around the building, the parameters, $\beta_{\boldsymbol{k}}, \tau_{\boldsymbol{k}}$, and $\theta_{k}$ are, randomly time-varying functions. However, the rate of their variations is very slow compared to any useful signaling rates that are likely to be considered. It follows from (20) and (21) that the received signal, which is the time convolution of $x(t)$ and, $h(t)$ is given by

$$
y(t)=\sum_{k} \beta_{k} p\left(t-\tau_{k}\right) e^{j \theta_{k}\left[\omega\left(t-\tau_{k}\right)+\emptyset+\theta_{k}\right]}
$$

This signal is picked up by receiving antenna and amplified by low noise amplifier, the signal detected by square law envelope detector can be given to CRO. The power profile becomes 
$|y(t)|^{2}=\sum_{k} \sum_{l}\left\{\beta_{k} \beta_{l} p\left(t-\tau_{k}\right) p\left(t-\tau_{l}\right) e^{j\left[\theta_{k}-\theta_{l}+\omega\left(\tau_{l}-\tau_{k}\right)\right]}\right\}$

If there were no overlap of pulses, then (23) would have reduced to

$$
|y(t)|^{2}=\sum_{k} \beta_{k}^{2} p^{2}\left(t-\tau_{k}\right) \text {, no overlap }
$$

which is much simpler to use than (4) in estimating the $\beta$ 's and the $\tau$ 's from the measured $|y(t)|^{2}$ waveform.

$$
E_{\theta}\left\{|y(t)|^{2}\right\}=\sum_{k} \beta_{k}^{2} p^{2}\left(t-\tau_{k}\right)
$$

which is identical to (5) but allows for pulse overlap.

if the total swept bandwidth $\Delta f$ is sufficiently large, it can be shown using (23)

$$
s(t) \equiv \frac{1}{\Delta f} \int_{\frac{\Delta f}{2}}^{\frac{\Delta f}{2}}|y(t)|^{2} d f \approx \sum_{k} \beta_{k}^{2} p^{2}\left(t-\tau_{k}\right)
$$

$\Delta f=200 \mathrm{MHz}$, which is sufficient to satisfy (26) for pulses that are separated by more than about 4 ns. Thus, overlapping pulses of closer separation could not be resolved Given the frequency averaged power profile and knowing the shape of transmitted pulse $p(t), \tau_{k}$ and $\beta_{k}^{2}$ can be found. This model starts with physical realization that rays arrives in clusters. The cluster arrival times are modeled as Poisson arrival process with some fixed rate $\Lambda$, Within each cluster subsequent rays also arrives according to Poisson process with another fixed rate $\lambda$. Each cluster consists of rays i.e. $\lambda \ll \Lambda$.

Let arrival time of $l^{\text {th }}$ cluster be $T_{l}, l=0,1,2, \ldots$.

Let the arrival time of $k^{\text {th }}$ ray measured from beginning of the $l^{\text {th }}$ cluster be $\tau_{k, l}, k=0,1,2, \ldots$

For the first cluster $T_{0}=0$ and for the first ray within $l^{\text {th }}$ cluster $\tau_{k, l}=0$.

Hence $\tau_{k, l}$ and $T_{l}$ are independent interarrival exponential probability density function.

$$
\begin{gathered}
p\left(T_{l} / T_{l-1}\right)=\Lambda \exp \left[-\Lambda\left(T_{l}-T_{l-1}\right)\right], l>0 \\
p\left(\tau_{k, l} / \tau_{(k-1), l}\right)=\lambda \exp \left[-\lambda\left(\tau_{k, l}-\tau_{(k-1), l}\right)\right], k>0, l>0
\end{gathered}
$$

The amplitudes of $k^{\text {th }}$ path within the $l^{\text {th }}$ cluster obeys Rayleigh distribution. Whereas the clustering of the multipath arrivals, S-V model uses two Poisson process to describe multipath channel. The first Poisson process describes the arrival of the cluster, and second process describes the arrival of rays within that cluster.

$T_{l}$ : the arrival time of the first path of the $l^{\text {th }}$ cluster.

$\tau_{k l}$ : the delay of the $k^{\text {th }}$ path within $l^{\text {th }}$ cluster relative to the first path arrival time. $\Gamma$ : cluster arrival rate.

$\lambda$ : ray arrival rate $i . e$. the arrival rate of path within each cluster. Therefore, $\tau_{0 l}=T_{l}$. The distribution of cluster arrival time and ray arrival time are given by (27), (28).

Let the gain of $k^{\text {th }}$ ray of $l^{\text {th }}$ cluster be denoted by $\beta_{k, l}$ and its phase $\theta_{k, l}$. Hence impulse response given by (21) will become

$$
h(t)=\sum_{l=0}^{\infty} \sum_{k=0}^{\infty} \beta_{k l} e^{j \theta_{k l}} \delta\left(t-T_{l}-\tau_{k l}\right)
$$

$\theta_{k l}$ is statistically independent uniform random variable and $\beta_{k l}$ is statistically independent positive random variable.

The IEEE group made some modification on S-V channel using log-normal distribution to express multipath amplitudes and using another log-normal stochastic variable to express general multipath fluctuations.

Mathematically, the impulse response is described as (30)

$$
h_{i}(t)=X_{i} \sum_{l=0}^{L} \sum_{k=0}^{K} \alpha_{k, l}^{i} \delta\left(t-T_{l}^{i}-\tau_{k, l}^{i}\right)
$$

where $\left\{\alpha_{k, l}^{i}\right\}$ are the multipath gain coefficients, $\left\{T_{l}^{i}\right\}$ is the delay of the $l^{\text {th }}$ cluster, $\left\{\tau_{k, l}^{i}\right\}$ is the delay of the $k^{\text {th }}$ multipath component relative to the $l^{\text {th }}$ cluster arrival time, $\left\{X_{i}\right\}$ represents the log-normal shadowing, and $i$ refers to the $i^{\text {th }}$ realization. The distribution of cluster arrival time and the ray arrival time are given by (27) and (28). The channel coefficients are defined as a product of small-scale and large-scale fading coefficients, i.e. (28)

$$
\alpha_{k, l}=P_{k, l} \xi_{l} \beta_{k, l}
$$

The amplitude statistics of the measurements were found to best fit the log-normal distribution rather than the Rayleigh that was used in the original S-V model. In addition, the large-scale fading is also log-normally distributed.

$$
\begin{aligned}
& 20 \log 10\left(\xi_{l} \beta_{k, l}\right) \propto \operatorname{Normal}\left(\mu_{k, l}, \sigma_{1}^{2}+\sigma_{2}^{2}\right) \\
& \text { Or }\left|\xi_{l} \beta_{k, l}\right|=10^{\frac{\left(\mu_{k, l}+n_{1}+n_{2}\right)}{20}}
\end{aligned}
$$

where $n_{1} \propto \operatorname{Normal}\left(0, \sigma_{1}^{2}\right)$ and $n_{2} \propto \operatorname{Normal}\left(0, \sigma_{2}^{2}\right)$ are independent and correspond to the fading on each cluster and ray, respectively. The behavior of the power delay profile is

$$
E\left[\left|\xi_{l} \beta_{k, l}\right|^{2}\right]=\Omega_{0} \exp \left(\frac{-T_{l}}{\Gamma}\right) \exp \left(\frac{-X_{k, l}}{\gamma}\right)
$$

which reflects the exponential decay of each cluster, as well as the decay of the total cluster power with delay.

The $\mu_{k, l}$ is given by

$$
\mu_{k, l}=\frac{10 \ln \left(\Omega_{0}\right)-10 T_{l} / \Gamma-10 \tau_{k, l} / \gamma}{\ln (10)}-\frac{\left(\sigma_{1}^{2}+\sigma_{2}^{2}\right) \ln (10)}{20}
$$

In the above equations, $\xi_{l}$ reflects the fading associated with the $l^{\text {th }}$ cluster and $\beta_{k, l}$ corresponds to the fading associated with the $k^{t h}$ ray of the $l^{\text {th }}$ cluster. The distribution of cluster arrival time and ray arrival time are given by (27) and (28). The parameters are given by $\Gamma$ : cluster decay factor, $\gamma$ : ray decay factor, $\sigma$ : standard deviation of log-normal fading term $(\mathrm{dB})$.

Afterwards, this model was modified by IEEE group which has some problems: S-V/IEEE802.15.3a can exactly reflect the propagation law of indoor NLOS environment, but its capability of indoor LOS environment is insufficient. Moreover this model cannot exactly predict multipath propagation characteristics of indoor conditions.

\section{MODEL PARAMETERS}

\section{A. Cluster arrival rate}

When the wave is transmitted it reaches to the receiver through more or less direct path. This path is usually straight lines in open space and hallways. This is the first cluster appearing at the receiver. The signal may undergo reflections because of furniture, wall, obstacles and other moving bodies in the room which is then picked up by receiver. Hence this results in subsequent clusters. The first cluster is always present while others may or may not present (Fig.3). Using Poisson distribution

$$
p\left(T_{l} / T_{l-1}\right)=\Lambda \exp \left[-\Lambda\left(T_{l}-T_{l-1}\right)\right], l>0
$$

It is possible to estimate clusters which have probability $\mathrm{P}(0), \mathrm{P}(1), \mathrm{P}(2) \ldots \mathrm{P}(\mathrm{n})$.

\section{B. Ray arrival rate $\lambda$}

Within a cluster, the ray arrivals are also described by a Poisson process, so that the distribution of the inter-arrival times is also an exponential random variable for $k>0$

$$
p\left(\tau_{k, l} / \tau_{(k-1), l}\right)=\lambda \exp \left[-\lambda\left(\tau_{k, l}-\tau_{(k-1), l}\right)\right], k>0, l>0
$$

where $\lambda$ is the mean ray arrival rate. If arrival time of the 
first cluster be reference time, then $T_{1}^{i}=0$, we have

$$
T_{2}^{i}-T_{1}^{i}=T_{2}^{i}=T_{N}
$$

where $T_{N}$ is time delay between two clusters, hence $\tau_{0,1}=0,(m=1,2)$

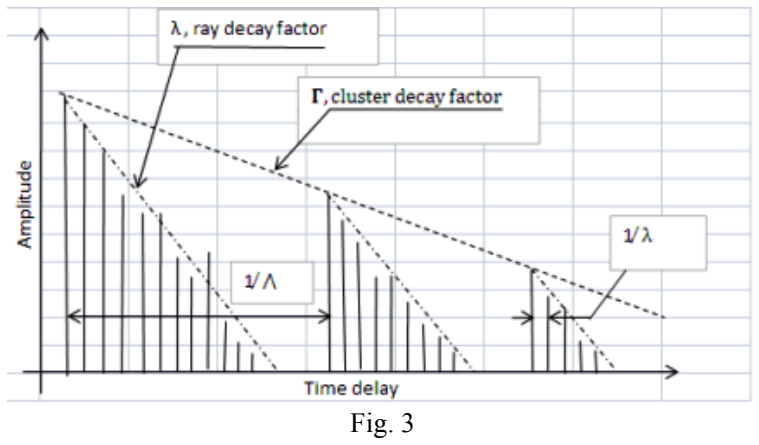

The rays within cluster are modeled as Poisson arrival process. Thus distribution of ray arrival time within each cluster is

$$
\begin{aligned}
& p\left(\tau_{k, m} \mid \tau_{(k-1), m}\right)=\lambda \exp \left[-\lambda\left(\tau_{k, m}-\tau_{(k-1), m}\right)\right], \\
& m=1,2
\end{aligned}
$$

The average power of both the clusters and the rays within the clusters are assumed to decay exponentially such that the average power of a multi-path component at a given delay $T_{l}=\tau_{k, l}$, given by

$$
\overline{\beta_{k, l}^{2}}=\overline{\beta_{0,0}^{2}} \exp \left(-\frac{T_{l}}{\Gamma}\right) \exp \left(-\frac{\tau_{k, l}}{\gamma}\right)
$$

where $\overline{\beta_{0,0}^{2}}$ is the expected value of the power of the first arriving multi-path component, $\Gamma$ is the decay exponent of the clusters and $\gamma$ is the decay exponent of the rays within a cluster. The magnitudes of the path amplitudes are assumed to follow a lognormal distribution about the expected value with standard deviation. The polarity of each path is represented as an equi-probable binary random variable. One of the key disadvantages of the Saleh-Valenzuela model is that channel impulse responses generated using this model display a large amount of "visible" clustering. Figure 1 shows the average amplitude of measured channel impulse responses at a single location. We see that the measured channel impulse responses do not display significant clustering as opposed to the Saleh-Valenzuela model, even if the small scale statistics of the two are matched. Therefore the channel impulse responses generated using the Saleh-Valenzuela model do not "resemble" the measured channel impulse responses even if the small-scale statistics (such as RMS delay spread, mean excess delay and the number of significant multipath) are similar.

\section{Large scale fading}

It is defined as the variation of the local mean around the path gain. It is commonly modeled as a lognormal distribution, with a variance of typically $1-2 \mathrm{~dB}$ (LOS) and 2-6 dB (NLOS), depending on the environment [14]. The authors in [15] suggested modeling the shadowing variance as random variable where the distribution (from house to house) is lognormal. The total attenuation due to shadowing and path gain (in $\mathrm{dB}$ ) is thus

$\left[-G_{p r, 1}+10 \log (d)\right]+\left[10 n_{1} \sigma_{\gamma} \log _{10} d+n_{2} \mu_{\sigma}+n_{2} n_{3} \sigma_{\sigma}\right]$ where $n_{1}, n_{2}$ and $n_{3}$ are zero-mean, unit-variance Gaussian variables.

The large scale fading is related to diffraction and reflection effects that the MPCs undergo on their way between TX and RX, so that one could anticipate a frequency dependence of the shadowing. However, measurements in[16] indicate that the shadowing variance is approximately independent of frequency.

\section{Path arrival times}

For interarrival times of MPC's within clusters number of models are proposed. Regularly spaced arrival times which is suitable for dense channel models. Poisson arrival times is the most popular for arrival times within clusters.

$$
p\left(\tau_{k, l} / \tau_{(k-1), l}\right)=\lambda \exp \left[-\lambda\left(\tau_{k, l}-\tau_{(k-1), l}\right)\right], k>0, l>0
$$

where by definition $\tau_{0, l}=0$. However some UWB measurement indicate that arrival rate is larger for later clusters. Mixed Poisson process suggests mixture of two Poisson processes [22].

$$
\begin{aligned}
& p\left[\tau_{k, l} \mid \tau_{(k-1), l}\right]=\beta \lambda_{1} \exp \left[-\lambda_{1}\left(\tau_{k, l}-\tau_{(k-1), l}\right)\right]+(1- \\
& \beta) \lambda_{2} \exp \left[-\lambda_{2}\left(\tau_{k, l}-\tau_{(k-1), l}\right)\right]
\end{aligned}
$$

\section{E. Cluster power}

The most common model for the power delay profile of each cluster is a one-sided exponential decay

$$
E\left\{\left|a_{k, l}\right|^{2}\right\} \propto \Omega_{\operatorname{lexp}\left(-\tau_{k, l} / \gamma_{l}\right)}
$$

where $\Omega_{l}$ is the integrated energy of the $l^{\text {th }}$ cluster, and $\gamma_{l}$ is the intracluster decay time constant. The cluster powers, averaged over the large scale fading, in general follow an exponential decay

$$
10 \log \left(\Omega_{l}\right)=10 \log \left[\exp \left(-T_{l} / \Gamma\right)\right]
$$

The intercluster decay time constant $\Gamma$ is typically around 10-30 ns. while widely differing values (between 1 and $60 \mathrm{~ns}$ ) have been reported for the intracluster constant $\gamma$ [17]. In equation (27), $1 / \Lambda$ is typically in the range of $10-50 \mathrm{~ns}$. It is not only quite simple, but also agrees well with many measurements, and is thus widely used. Still, it does not account for some experimentally observed facts 1) The first component of a cluster can show a stronger power than the one given by (18). In conventional narrowband modeling, such a strong first component usually occurs only in the first cluster, and can be interpreted as a line of sight connection. Several UWB measurements have shown strong components for later clusters, probably due to specular reflections.

2) The cluster decay rates $\gamma_{l}$ depend on the delay of the cluster. A possible solution is to prescribe a linear increase of $\gamma_{l}$ with the cluster delay.

$\gamma_{l} \propto T_{l}+\gamma_{0}$, where $k_{\gamma}$ and $\gamma_{0}$ are constants. The decay time constants also show a dependence on the distance. Note that the sum of the cluster powers is the total power, and thus the cluster power variations also determine the variance of the shadowing. In some environments, the cluster shape does not show a sharp onset, but rather first a gradual increase until a local maximum is reached, and then a decrease. It must be stressed that the extraction of $\mathrm{S}-\mathrm{V}$ parameters from measured data is a rather ambiguous procedure. It is often an arbitrary decision whether to consider a power delay profile as a superposition of several closely spaced clusters, or as a single cluster. Naturally, this has a great impact on the number of clusters as well as the inter-cluster decay time constants. 


\section{SUMmary OF S-V MODIFIED CHANNEL}

From the discussions of preceding sections we can see that the clustering of the multipath arrivals was observed in the time-domain indoor multipath propagation channel. Each MPC's arrive at receiver in clusters, and each cluster includes many single signals. It is correlated with the time-of-arrival and angle-of-arrival of the multipath components. The channel model can be described in an impulse response expression,

$$
h(t, \theta)=\sum_{l=0}^{L} \sum_{k=0}^{K} \beta_{k, l} e^{j \theta_{k l}} \delta\left(t-T_{l}-\tau_{k, l}\right)
$$

which is correlated with the time-of-arrival and angle-of-arrival. If the arrival time and arrival angle of the multipath components have dependency, then evaluation of the cluster mean square angle will lead to longer delay. However, from the measurements [20], it was showed that the channel characteristics dissatisfied this point. Due to the independency of the time and angle completeness impulse response sequence $h(t, \theta)$ can be $h(t, \theta)=h(t) h(\theta)$ in this way, $h(\theta)$ is absolutely independent. Based on the S-V model, the new $h(t)$ can be described as

$$
h(t)=\sum_{l=0}^{L} \sum_{k=0}^{K} \beta_{k, l} \delta\left(t-T_{l}-\tau_{k, l}\right)
$$

$h(\theta)$ is an independent angle impulse response, it can be considered as a model which is similar to the channel time impulse response, and the model is given by

$$
h(\theta)=\sum_{l=0}^{L} \sum_{k=}^{K} \delta\left(\theta-\omega_{k l}\right)
$$

$\omega_{k l}$ is the arrival angle of the $k^{t h}$ path within the $l^{t h}$ cluster. From the measurement channel pictures [21], the signal amplitude can be observed fading to zero with the temporal change. Therefore, this model can best fit the channel characteristics if $\omega_{k l}$ obeys Laplacian distribution. So suppose the mean value of $\omega_{k l}$ is zero, the standard deviation is $\sigma$, its probability density is described as

$$
p(\omega)=\frac{1}{\sqrt{2} \sigma} e^{-|\sqrt{2} \omega / \sigma|}
$$

\section{Simulation Result And Analysis}

Here we describe different channel models which are commonly used for wireless communication in indoor applications. From the survey it seems that different authors presented their work where there are large differences in channel models. We note that there is no universal stochastic model of wireless propagation. It is observed that clustering is common phenomenon. Saleh- Valenzuela and Suzuki did major work in area. IEEE group modified S-V model and is best model which fit to UWB physical channel model. Hence with the help of standard S-V model we can check the capabilities of physical model.

Here we use MATLAB to simulate channel model for continuous and discrete time channels. Simulation is done for residential, industrial and outdoor environment for both LOS and NLOS. It is observed that propagation is complex in indoor environment because of furniture, machines, metal objects and motion of the people. Hence signal appear at receiver in clusters with decaying power.

UWB channel is quassi-static because of motion of people, but this variation is negligible as compared to signal speed.

Fig. 5 shows power is decaying exponentially. The decay time constant of exponential function seem to follow log-normal distribution. In order to compare different multipath channel, the temporal dispersion parameters are used. We calculated the $\tau_{r m s}, \tau_{m}$ and number of multipaths at threshold level of $10 \mathrm{~dB}$ and $85 \%$ level. Because of temporal dispersion the signal received at receiver will distorted because of this spread. This will cause intersymbol interference which appears in communication system.

We compare our result with IEEE 802.15.3a and shows that results are perfect. The predicted values for a distance of 4 to 12 meters are $\Gamma=18 \mathrm{~ns}, \Lambda=0.0667, \gamma=12 \mathrm{~ns}$, $\lambda=3 n s$.

The frequency range involved in this simulation is in the range of $6 \mathrm{GHz}$ to $8 \mathrm{GHz}$. In table 1 and 2 the results for residential LOS are given for different cluster arrival rates from 0.010 to 0.14 and channel characteristics are found. Results are as per prediction. As cluster arrival rate increases, the $\tau_{r m s}, \tau_{m} N P_{10 d B}$ and $N P_{85 \%}$ increaes.
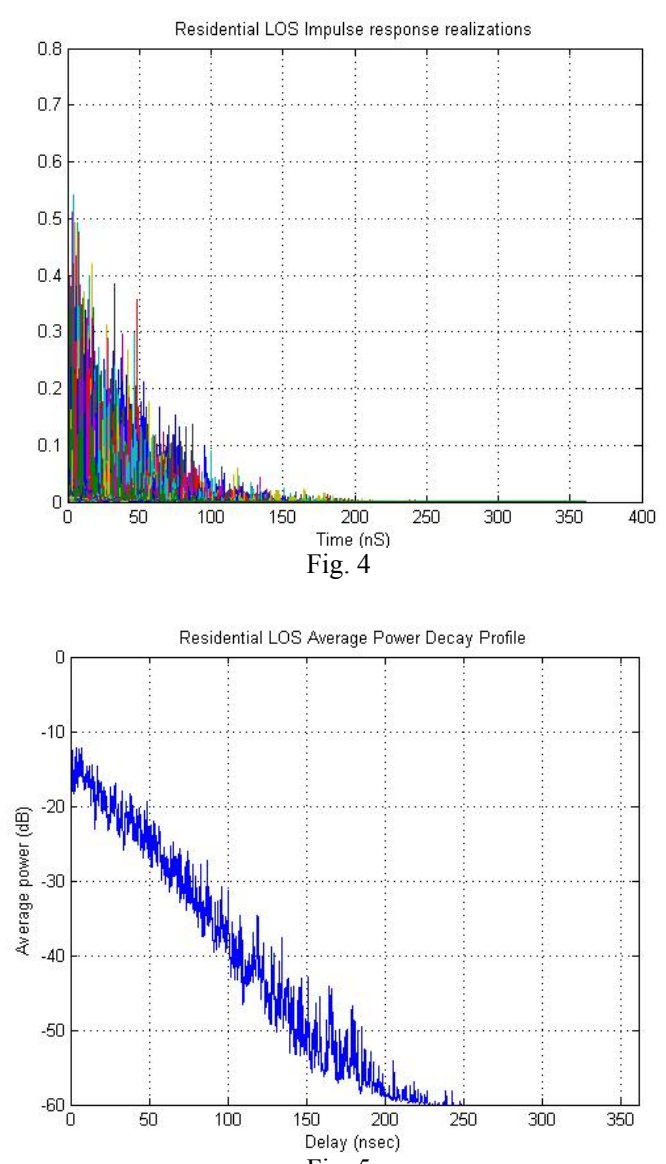

Fig. 5

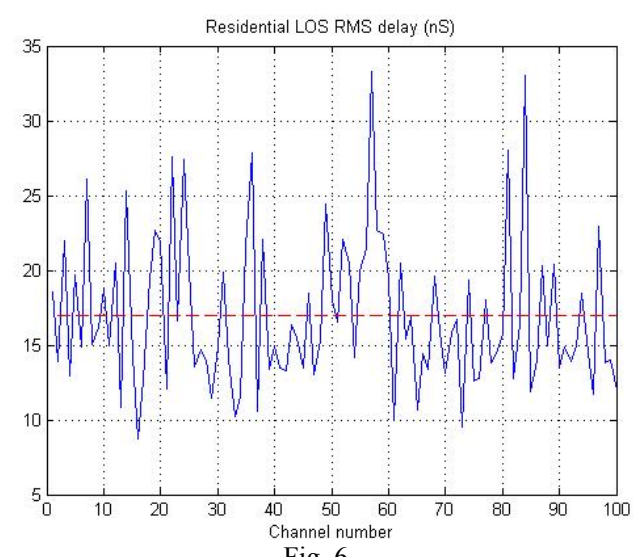

Fig. 6 


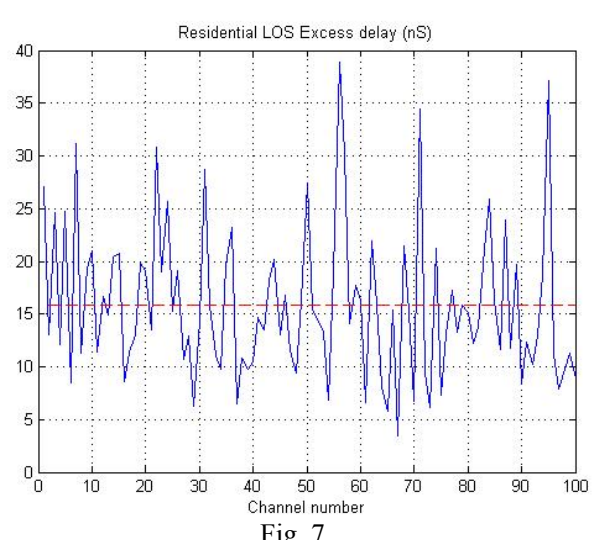

Fig. 7

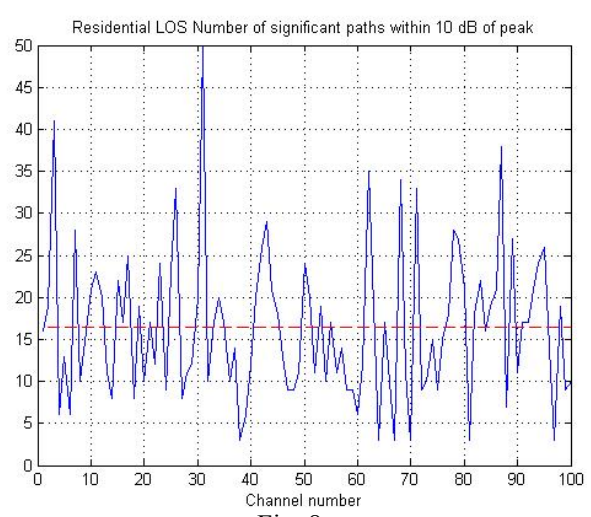

Fig. 8

Table I:

LOS Residential

Channel Parameters Cluster arrival rate : $0.01-0.14$

\begin{tabular}{|l|l|l|l|l|l|l|l|l|} 
Gama & Lmean & Lambda 1 & Lambda 2 & Sigma cluster & Beta & fc & fs \\
\hline
\end{tabular}

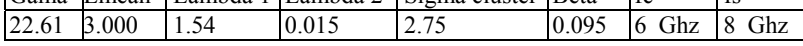

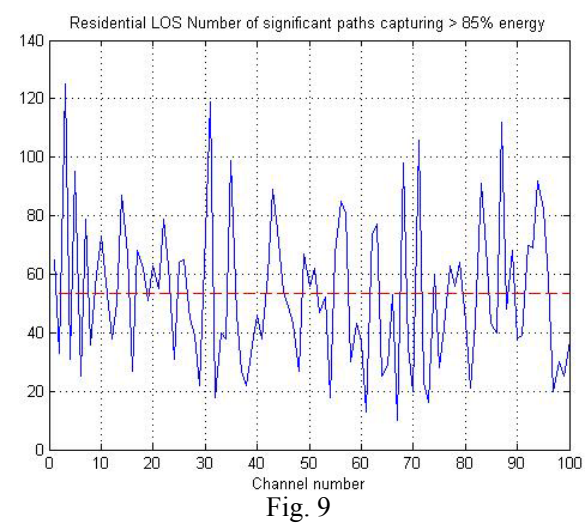

Table II:

Channel Characteristics

\begin{tabular}{|c|l|l|l|l|l|l|l|}
\hline$\Lambda$ & 0.01 & 0.03 & 0.05 & 0.07 & 0.09 & 0.12 & 0.14 \\
\hline$\tau_{r m s}$ & $18 \mathrm{~ns}$ & $18 \mathrm{~ns}$ & $17 \mathrm{~ns}$ & $16 \mathrm{~ns}$ & $15 \mathrm{~ns}$ & $15 \mathrm{~ns}$ & $14 \mathrm{~ns}$ \\
\hline$\tau_{m}$ & $13.7 \mathrm{~ns}$ & $15.7 \mathrm{~ns}$ & $15.9 \mathrm{~ns}$ & $15.6 \mathrm{~ns}$ & $15.2 \mathrm{~ns}$ & $14.7 \mathrm{~ns}$ & $14.4 \mathrm{~ns}$ \\
\hline$N P_{10 \mathrm{~dB} B}$ & 12 & 14.8 & 16.7 & 17.9 & 19.8 & 19.8 & 20.1 \\
\hline$N P_{85 \%}$ & 37.6 & 49.2 & 53.7 & 56.6 & 58.9 & 59.8 & 59.2 \\
\hline
\end{tabular}

Table III:

Channel Parameters

$\Gamma$ Cluster Decay Factor : $10-70$

\begin{tabular}{|l|l|l|l|l|l|l|l|}
\hline Lam & Lmean & $\begin{array}{l}\text { Lambda } \\
1\end{array}$ & $\begin{array}{l}\text { Lambda } \\
2\end{array}$ & $\begin{array}{l}\text { Sigma } \\
\text { cluster }\end{array}$ & Beta & fc & fs \\
\hline 0.047 & 3.000 & 1.54 & 0.015 & 2.75 & 0.095 & $6 \mathrm{Ghz}$ & $8 \mathrm{Ghz}$ \\
\hline
\end{tabular}

Table IV:

Channel Characteristics

\begin{tabular}{|c|l|l|l|l|l|l|l|}
\hline$\Gamma$ & 10 & 20 & 30 & 40 & 50 & 60 & 70 \\
\hline$\tau_{r m s}$ & $14 \mathrm{~ns}$ & $17 \mathrm{~ns}$ & $18 \mathrm{~ns}$ & $20 \mathrm{~ns}$ & $20 \mathrm{~ns}$ & $21 \mathrm{~ns}$ & $21 \mathrm{~ns}$ \\
\hline
\end{tabular}

\begin{tabular}{|c|l|l|l|l|l|l|l|}
\hline$\tau_{m}$ & $12.2 \mathrm{~ns}$ & $15.3 \mathrm{~ns}$ & $17.9 \mathrm{~ns}$ & $20 \mathrm{~ns}$ & $21.6 \mathrm{~ns}$ & $22.9 \mathrm{~ns}$ & $23.9 \mathrm{~ns}$ \\
\hline$N P_{10 \mathrm{~dB}}$ & 14.1 & 16.2 & 17.6 & 18.6 & 19.3 & 19.8 & 20 \\
\hline$N P_{85 \%}$ & 43.9 & 52.1 & 57 & 60.3 & 62.4 & 63.8 & 64.8 \\
\hline
\end{tabular}

Table V:

Channel Parameters
\begin{tabular}{|l|l|l|l|l|l|}
$\begin{array}{l}\text { Channel } \\
\text { number }\end{array}$ & Description & $\begin{array}{l}\text { Mean excess } \\
\text { delay }\end{array}$ & $\begin{array}{l}\text { Rms } \\
\text { delay }\end{array}$ & $\begin{array}{l}\text { \# path NP } \\
10 \mathrm{~dB}\end{array}$ & $\begin{array}{l}\text { \# path NP } \\
100 \%\end{array}$ \\
\hline 1 & Residential LOS & $15.9 \mathrm{~ns}$ & $17 \mathrm{~ns}$ & 16.5 & 53.3 \\
\hline 2 & Residential NLOS & $21.1 \mathrm{~ns}$ & $20 \mathrm{~ns}$ & 39.9 & 121.7 \\
\hline 3 & Office LOS & $9.2 \mathrm{~ns}$ & $9 \mathrm{~ns}$ & 15.1 & 21.8 \\
\hline 4 & Office NLOS & $16.2 \mathrm{~ns}$ & $13 \mathrm{~ns}$ & 26.8 & 42.8 \\
\hline 5 & Outdoor LOS & $27.5 \mathrm{~ns}$ & $29 \mathrm{~ns}$ & 18.2 & 35.8 \\
\hline 6 & Outdoor NLOS & $83.9 \mathrm{~ns}$ & $83 \mathrm{~ns}$ & 21.4 & 84 \\
\hline 7 & Industrial LOS & $1.6 \mathrm{~ns}$ & $8 \mathrm{~ns}$ & 7.3 & 9.4 \\
\hline 8 & Industrial NLOS & $24.1 \mathrm{~ns}$ & $20 \mathrm{~ns}$ & 132.4 & 184.5 \\
\hline 9 & $\begin{array}{l}\text { Open outdoor } \\
\text { NLOS }\end{array}$ & $18.9 \mathrm{~ns}$ & $23 \mathrm{~ns}$ & 5.7 & 6.9 \\
\hline
\end{tabular}

Table VI:

Comparison
\begin{tabular}{|c|l|l|}
\hline Channel characteristics & $\begin{array}{l}\text { Results from } \\
\text { IEEE } 802.15 .3 \text { a Model }\end{array}$ & $\begin{array}{l}\text { Results from our } \\
\text { Model }\end{array}$ \\
\hline $\begin{array}{l}\text { Mean excess delay } \\
\text { (nsec) } \tau_{m}\end{array}$ & 21.198 & 20.10 \\
\hline Rms delay (nsec) $\tau_{r m s}$ & 19.835 & 17.21 \\
\hline$N P_{10 d B}$ & 50.840 & 52.11 \\
\hline$N P_{85 \%}$ & 99.86 & 99.01 \\
\hline
\end{tabular}

\section{CONCLUSION}

In this paper, characteristics of UWB channels are analyzed based on simulations performed in various indoor/outdoor environments for both LOS and NLOS scenarios. A statistical model for the propagation of UWB signals in an indoor LOS channel was generated as a modification of SV/ IEEE 802.15.3a model. The model has a channel impulse response of a fast decaying cluster.

It means that the proposed model is more accurate in modeling the small scale characteristics of our set of indoor/outdoor LOS/NLOS environment measurement data. It has been noted that the geometry of the situation and the building architecture can have a significant effect on the received signals. Therefore, further work remains in the collection and processing of propagation data from different buildings, to increase the significance of and augment the results presented in this work. It is possible therefore, to apply our model to detect location of mobile user in indoor environment.

The simulation result shows that the proposed model outperforms the SV/ IEEE 802.15.3a model when considering CDFs for the channel statistics. It means that the proposed model is more accurate in modeling the small scale characteristics of our set of indoor LOS environment measurement data. With this model, accurate performance predictions of UWB systems become feasible.

\section{REFERENCES}

[1] D. C. Cox, "Universal Portable Rdio Communication" IEEE Trans. Veh, Techno. Vol. VT-34, pp 117-121, Aug. 1985.

[2] D. C. Cox, H. W. Armold and P. T. Porter, "Universal portable digital communication : A system perspective" IEEE J. selected areas communication vol. SAC-5,pp. 764-773, June 1987.

[3] W. C. Jakes, Microwave Mobile Communication, New York, Wiley, 1974.

[4] S. E. Alexander,"Radio propagation within building at $900 \mathrm{Mhz}$ Electron Letter, vol.18, No. 21, pp. 913-914, Oct , 1982. 
[5] H. H. Hoffman and D. C. Cox,"Attenuation of $900 \mathrm{Mhz}$ radio waves propagating into metal building" IEEE Trans. Antenna Propagation, vol. AP-30, pp. 808-811, July, 1982.

[6] D. M. J. Devasirvatham,"The delay spread measurements of wide band radio signals within building" Electron Letter, vol. 20 No. 23, pp. 950-951, Nov, 1984

[7] Molish A. F. " Ultra-wideband propagation channel theory, measurement and modeling" IEEE Trans. Veh. Tech. 2005,54(5), $1528-1545$.

[8] A. B. Carlson, Paul B. Crilly, "Communication Systems" McGraw-Hill International Edition, 2010.

[9] I. Oppermann , M. Hamalainen and J. Iinatti, "UWB Theory and Application" John Willy and Sons, West Sussex, 2004.

[10] H. Suzuki, "A statistical model for urban radio propagation" IEEE Trans. On Commn. Vol.com-25, No. 7, July, 1977.

[11] Theodore S. Rappaport, "Wireless Communicationd", Prentice-Hall of India, 2007.

[12] Adel A. M. Saleh and Reinaldo A. Valenzuela " A statistical model for indoor multipath propagation"IEEE Journ. Select. Area Commn. Vol. SAC-5 No. 2, pp. 128-137, Feb. 1987.

[13] L. Ge, G. Yue and S. Affes,"On the BER performance of pulse position modulation UWB radio in multipath channels" Proc. Of IEEE on UBW Systems and Technologies, pp. 231-234, May, 2002

[14] B. Kannan et al, "UWB Channel Charactrization in office environments" IEEE Tech. Rep. Document, IEEE802.15-04-0439-00-004a, 2004

[15] S. S. Ghassemzadeh, R. Jana, C. W. Rice, W. Turin and V. Tarokh "Measurement and modelling of UWB indoor channel" IEEE Trans. Commn. Vol.52, pp. 1786-1796, 2004.

[16] J. K. Kederal, S. Wyne, P. Almers, F. Tufvesson and A. F. Molish, "Statistical analysis of the UWB channel in industrial environment" In Proc. Of IEEE VTC, Fall, 2004, pp. 81-85.

[17] B. Kannan et al, "UWB Channel Charactrization in office environments" IEEE Tech. Rep. Document, IEEE802.15-04-043900-004a, 2004.
[18] J. R. Foerster Q. Li, "UWB channel modelling contribution from Intel," IEEE P802.15, Working Group for Personal Area Networks, June, 2002.

[19] R. Buehrer,W. Davis, A. Safaai-Jazi and D. Sweeney, " Ultra-wide band Propagation Measurements and Modeling - Final Report to DARPA NETEX Program" Virginia Tech.rep. 2004.

[20] Quentin Spencer, Michael Rice, Brian Jeffs and Michael Jensen "Indoor Wideband Timr/Angle of Arrival multipath propagation Results" IEEE o-7803-3659-3/97, pp. 1410-1414, 1997.

[21] R. A. Scholtz, R. J. Cramer, M. Z. Win " Evaluation of Propagation Characteristics of UWB Commn. Channels" IEEE, Ant. and Propa. Soc. Symp. 1998.

[22] D. J. Clabaugh, M. A. Temple, R. A. Raines and C. M. Canadeo,’UWB multiple access performance using time hopped pulse position modulation with biorthogonal signalling" IEEE Conf: UWB Systems and Technologies, Dig. Papers, Restor, V A Nov. 2003, pp. 330-333.

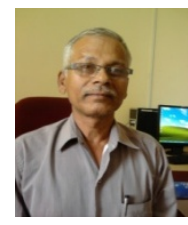

Mr, B. R. Jadhavar received M. E.(E\&T/C), degree from College of Engineering Pune affiliated to University of Pune in June, 2001. He is research student in Shree Guru Govind Singhji Institute of Engineering and Technology, Nanded, affiliated to SRTM University, Nanded. His research interests are mobile user location in indoor wireless system in microwave frequency band. Currently he is working as Assistant Professor in Siddhant College of Engineering, Pune (India)

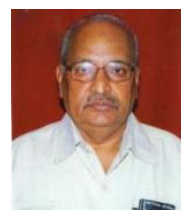

T.R. Sontakke passed B. E. (Elect ) in June 1971, from Govt . College of Engineering Aurangabad and M.Tech. in June 1973, from Nagpur University with specialization in power systems. He did $\mathrm{Ph}$. D from Indian Institute of Technology Mumbai, in 1980. He is now working as Principal of Siddhant College of Engineering, Pune(India). 